\title{
Rechtswissenschaft als Anthropotechnik: Bioethik und Menschenwürde ${ }^{1}$
}

Für die humanwissenschaftliche und ethische Diskussion von Problemen, die mit den Biowissenschaften und Reproduktionstechnologien zusammenhängen, ist die rechtswissenschaftliche Auseinandersetzung mit dem Begriff Menschenwürde besonders wichtig, weil das Recht für die Gesellschaft sowohl materiell wirksam wird, als auch auf etablierte Symboliken zurückgreift und neue Symbole schafft. Diese Zusammenhänge sollen im Folgenden diskutiert werden. Zunächst wird das Recht als Anthropotechnik eingeordnet. Als in sich scheinbar geschlossenes Ordnungssystem generiert es aufgrund seiner Wirkmacht Begrifflichkeiten und von hier aus Wirklichkeitsbilder, wobei die Nähe moderner rechtswissenschaftlicher Argumentationen zu einer Logik des Tabus auffällt (I). Daran anschließend wendet sich der zweite Abschnitt der performativen, symbol- und bildgebenden Qualität des Rechts zu (II). Schließlich wird der Umgang mit dem Begriff des Menschen und dessen Definition verfolgt, wobei im Vordergrund Fragen des Status der Geburt, der Identität von Embryo und »Mensch« sowie einer möglichen Offenheit des durch das Grundgesetz implementierten Menschenbildes stehen (III).

\section{Logik des Tabus}

Zu den wichtigsten Medien eines auf Kriterien der Menschenwürde rekurrierenden bioethischen Diskurses gehören Rechtswissenschaft und Rechtsprechung. Über Praktiken der Reflexion und der Urteilsfindung tragen sie zur bioethischen Debatte bei, indem sie diese zu kommentieren beabsichtigen, rechtliche Rahmenbedingungen und Grenzziehungen der Bioethik suchen oder indem sie bemüht sind, bioethische Überlegungen für Kontexte des Rechts fruchtbar zu machen. Die Schwierigkeit, die sich für die Rechtsprechung wie auch für rechtswissenschaftliche Beiträge ergibt, besteht vor allem darin, das Recht als objektiv funktionierenden Rahmen gesellschaftlicher Möglichkeiten zu betrachten, jedoch ebenso in der Reflexion auf die Bedingungen eines Instituts wie der Menschenwürde dessen Voraussetzungen thematisieren zu müssen. Vor diesem Hintergrund dient als Ausgangsthese dieses Aufsatzes die Annahme, daß das Recht, insofern es nicht allein Regeln und Begrenzungen für Individuen und Institutionen etabliert, sich als eine der effektivsten modernen Anthropotechniken bezeichnen läßt. So definiert, stellt die Praxis des Rechts selbst eine Technologie dar, mit deren Hilfe kulturelle Bedeutung produziert und gesellschaftliche Realität definiert wird. In diesem Sinne spricht Johannes Reiter davon, im 20. Jahrhundert habe sich ein universales Verständnis von Menschenwürde durchgesetzt: »Würde kommt dem Menschen bereits als Mitglied der Gattung Mensch zu. Das heißt, sie gilt für alle Menschen, ohne dass dafür erst bestimmte Leistungen erbracht oder bestimmte Qualitäten erfüllt wer-

1 Ich danke der Deutschen Forschungsgemeinschaft für ihre Unterstützung meiner Arbeit. 
den müssten. $\ll^{2}$ Die Liminalität des abstrakten Begriffspaares »Mensch« und »Menschenwürde« ergäbe sich somit aus der Summe derjenigen Lebensformen, die sich mit dem »Menschen« in Verbindung bringen ließen. Von primärem Interesse für ein normatives Institut, wie es die Menschenwürde darstellt, wäre dann gerade nicht deren abstrakter ethischer Gehalt, für den symbolische Transmitter wie der Begriff des »Menschen « gesucht werden, sondern umgekehrt die Summe einer Quantität an Lebensformen, deren Einhegung über die normativ liminale Institution erfolgt.

Das wird besonders deutlich, wenn Reiter die Kategorie der »Menschenwürde « als zentrales Medium in den Auseinandersetzungen um die »Stellung des Menschen in der technischen Zivilisation « bezeichnet. ${ }^{3}$ Die Aufnahme frühembryonaler Lebensformen in den Kontext von Grundrechts- und Menschenwürdeschutz ist nicht selbstverständlich. Erst der gemeinsame Zugriff von Legislative, Jurisprudenz und Ethik in der modernen Gesellschaft etikettiert jene Entitäten aus dem Zwischenreich von Mensch und Nichtmensch als »Embryonen«; mit dem Effekt, daß sie sich signifikant verwandeln. Aus lebendigen Dingen, die sich im Prozeß einer kontinuierlichen Entwicklung erst zum menschlichen Embryo und Fetus, dann zum Menschen befinden, werden »Menschen «, die, da sie Rechtspersonen sind, sich auch mit den Insignien von Rechtsschutz und menschlicher Würde ausgestattet sehen. ${ }^{4}$ Ein solches Verwandlungsgeschehen verweist auf eine arbiträre Liminalität des Menschen, die von wechselnden kulturellen Epistemologien und Genealogien abhängt - trotzdem gehört eine solche Liminalität offenbar zur kulturanthropologischen Definition des Menschen.

In dieser Perspektive ließe sich von einer magischen Kraft des Rechts sprechen, das lebendige Dinge zu Rechtssubjekten machen kann. Der Eintritt in die Rechtsordnung, nicht als deren Objekt, wie dies vornehmlich für Tiere und Dinge gilt, sondern als Subjekt derselben (und daher als Rechtsperson), nobilitiert dieses Subjekt in Richtung seiner Abhebung von der umgebenden Natur sowie als Mitglied einer menschlichen Gemeinschaft der Rechtssubjekte. Genau dies aber sei weder Pflanzen, Tieren, noch Embryonen möglich, dekretiert Norbert Hoerster und macht diesen Umstand am fehlenden »Überlebensinteresse« der genannten Entitäten fest, an der Fähigkeit Wünsche zu antizipieren und zukunftsfähig zu handeln. ${ }^{5}$ Allein Menschen, womit Hoerster wiederum nur geborene, mithin vernunftfähige Menschen meint, besäßen ein solches Interesse und wären demnach dazu prädestiniert, Rechtsgemeinschaften zu bilden und in solche aufgenommen zu werden. Gegen utilitaristische Positionen wie diese, suchen

2 Johannes Reiter: Menschenwürde als Maßstab, in: Aus Politik und Zeitgeschichte. Beilage zur Wochenzeitung Das Parlament, B 23-24/2004, S. $7 f$.

3 Ebd., S. 6.

4 Damit auch das bereits benannte Problem der Bildlichkeit berührend, weist Sheila Jasanoff zudem darauf hin, der rechtliche Status des Fetus stehe sich verändernden Konstellationen in der öffentlichen Wahrnehmungshaltung gegenüber: »The human fetus, until recently, was a largely invisible and voiceless member of society « (Sheila Jasanoff: Science at the Bar. Law, Science and Technology in America, Cambridge/MA \& London 1997, S. 161). Erst die technischen Innovationen der letzten Jahrzehnte hätten dem Fetus zunehmende physische Realität und wachsende Rechtsansprüche verschafft.

5 Vgl. Norbert Hoerster: Hat der Embryo wirklich ein Interesse am Leben, in: FAZ, vom 23. Juli 2001. 
Sigrid Graumann und Andreas Poltermann festzuschreiben, Menschenwürde sei nichts, was erworben werde und könne »auch wieder verloren gehen «. ${ }^{6}$ Verlieren läßt sich jedoch nur, was einmal hinzugewonnen wurde und nicht notwendig zur anthropologischen Ausstattung zählt. Das verdeutlicht einmal mehr die letztlich naturrechtliche Verankerung des Menschenwürdegedankens, der bioethisch in aller Regel als anthropologische Substanz formuliert ist, nicht etwa als kulturelles Artefakt.

Das Stadium einer bloßen Existenz in Natur (und damit auch, was wichtiger ist: als Natur) wird erst durch die Integration in einen rechtlichen Kontext beendet. In Übereinstimmung mit Max Weber konzediert Jürgen Habermas, erst in der Moderne habe sich »politische Herrschaft in Formen des positiven Rechts zur legalen Herrschaft entwickeln « können ${ }^{7}$ - womit diese sich von Formen einer normativen Autorität absetze, die sich aus dem sakralen, vorstaatlichen Recht generiere. ${ }^{8}$ Fraglich bleibt, ob mit der Etablierung positiven Rechts auch der sakrale bis magische Anteil an Recht und Normativität überwunden sei. So ordnet Jacques Derrida das Recht, gemeinsam mit der Politik und dem Sittlichen in den Kontext der »symbolischen Ordnung « ein und betont, auch das positive Recht - nicht etwa nur das Naturrecht - bleibe »der kritischen Befragung « entzogen, indem es sich von einer nur mystisch zu legitimierenden Gewalt herleite. ${ }^{9}$ Auch wenn positives Recht als Substitut des vorgängigen, magischen Rechtskosmos auftritt, muß es diesen nicht zwingend vollständig eliminieren; genauso gut könnte es ihn konsumieren und in der Folge partiell weiterhin von dessen Strukturelementen zehren. Indes meint Habermas, ein »nachmetaphysisches Recht «, beruhend auf der »reziproken Anerkennung kooperierender Rechtssubjekte«, löse bereits die vollständige Aufkündigung sakraler und magischer Anteile am Recht ein. ${ }^{10} \gg$ Denn als Rechtssubjekte«, erklärt er, »erlangen sie Autonomie erst dadurch, daß sie sich zugleich als Autoren der Rechte verstehen und betätigen, denen sie sich als Adressaten unterwerfen wollen. Die Idee der Selbstgesetzgebung muß sich im Medium des Rechts selbst Geltung verschaffen. $«{ }^{11}$ Das ist eine im Kern der kantischen Philosophie geschuldete Position, die gleichzeitig die sogenannte »Objektformel« Günther Dürigs über den Zweck des Rechts paraphrasiert. Worauf es aber ankommt, ist zum einen der darin angelegte performative Akt einer Verwandlung der Subjekte von einem Stadium ihrer (nicht autonomen) Existenz in ein anderes (als autonome Subjekte des Rechts) sowie zum anderen der dem Recht zugeschriebene Status als ein Medium, welches die Subjekte zu transzendieren in der Lage ist.

6 Vgl. Sigrid Graumann/Andreas Poltermann: Klonen: Ein Schlüssel zur Heilung oder eine Verletzung der Menschenwürde?, in: Aus Politik und Zeitgeschichte. Beilage zur Wochenzeitung Das Parlament, B 23-24/2004, S. 27.

7 Jürgen Habermas: Faktizität und Geltung. Beiträge zur Diskurstheorie des Rechts und des demokratischen Rechtsstaats, Frankfurt/M. 1992, S. 179; vgl.a. Max Weber: Wirtschaft und Gesellschaft, Tübingen 1980, S. 815-868.

8 Vgl. Jürgen Habermas: Faktizität und Geltung, a.a.O., S. 177.

9 Vgl. Jacques Derrida: Gesetzeskraft. Der »mystische Grund der Autorität«, Frankfurt/M. 1991, S. 70f.

10 Vgl. Jürgen Habermas: Faktizität und Geltung, a.a.O., S. 116.

11 Ebd., S. 160. 
In Hinblick auf magische Qualitäten des Rechts hat Émile Durkheim darauf hingewiesen, wie in archaischen Gesellschaften das Totem die Rolle einer Instanz als transzendenter Rechtsbewahrer und Rechtsbegründer zugleich wahrnimmt und sich Rechtsautorität über das Instrument des Tabus verschafft. ${ }^{12}$ Darauf referiert schließlich Sigmund Freud, indem er die These formuliert, es dürfe »uns ahnen [...], daß die Sitten- und Moralverbote, denen wir selbst gehorchen, in ihrem Wesen eine Verwandtschaft mit diesem primitiven Tabu haben könnten und daß die Aufklärung des Tabus ein Licht auf den dunklen Ursprung unseres eigenen >kategorischen Imperativs< zu werfen vermöchte. ${ }^{13}$ Freuds kulturtheoretische Synthese der Normen- und Rechtsproblematik läßt das moderne Recht in einem durch den Animismus zusätzlich aufgehellten Licht erscheinen, insofern die Rechtssubjekte die ihnen gesetzten Regeln natürlich nicht lediglich befolgen sollen, sondern von ihnen auch erwartet wird, das $»$ Sollen« der Rechtsnorm als verbindlich anzuerkennen.

Demzufolge trifft sich an diesem Punkt die Totemlogik des Rechts mit einem Kantianismus des Rechts, der ebenfalls bemüht ist, eine absolute Instanz der Rechtsbegründung zu implementieren. In diesem Sinne hat Hans Kelsen hervorgehoben: »Denn die Norm ist ein Sollen, der Willensakt, dessen Sinn sie ist, ein Sein. [...] Der Unterschied zwischen Sein und Sollen kann nicht näher erklärt werden. Er ist unserem Bewußtsein unmittelbar gegeben. « ${ }^{14}$ Wenngleich Kelsen eine absolute Moralisierung des Rechts ablehnt, bleibt das Problem der Normbegründung dennoch virulent. Da »der Geltungsgrund einer Norm [...] nur die Geltung einer anderen Norm « sein könne, ${ }^{15}$ wird es erforderlich, die daraus folgende, unendliche Iteration der Normsetzung an einem bestimmten Punkt abzubrechen. Dieses Problem löst Kelsen durch die Einführung der sogenannten »Grundnorm« als »höchste[r] vorausgesetzte[r] Norm«: die Normsetzung

12 Vgl. Émile Durkheim: Die elementaren Formen des religiösen Lebens, Frankfurt/M. 1994.

13 Sigmund Freud: Totem und Tabu, in: ders.: Studienausgabe Bd. IX, Frankfurt/M. 1974, S. 315. Wie als Illustration dieser von Freud entwickelten These, sowie flankierend zu der beschriebenen Abwehrhaltung gegenüber einer »technischen Zivilisation«, kann der Redebeitrag der Bundestagsabgeordneten Monika Knoche während der Beratung des Deutschen Bundestages zur Einsetzung einer Enquete-Kommission Recht und Ethik der modernen Medizin dienen. Während sie davon spricht, der Begriff des Menschen selbst sei »durch die Anwendung einer Technik [...] von seiner Auflösung bedroht«, schlußfolgert sie, in der Konsequenz würde »das Menschenrecht, das Projekt der Moderne, einer postmodernen Beliebigkeit« anheim gestellt (Deutscher Bundestag: Plenarprotokoll 14/96, Stenographischer Bericht, 96. Sitzung, Berlin, Freitag, den 24. März 2000: TOP 20: Beratung des Antrags zur Einsetzung einer Enquete-Kommission Recht und Ethik der modernen Medizin, BT-Drucksache 14/3011, Monika Knoche [BÜNDNIS 90/DIE GRÜNEN], http://www.bundestag.de/ bic/plenarprotokolle/pp/2000/index.htm, 21.12.2003). Die Verwendung des »Menschenrechts « im Singular wäre hier von großem Interesse, da es Menschenrechte nur im Plural gibt. Über dieses singulare Verständnis des Menschenrechts gelangt Knoche aber zu ihrer Kernthese: »Wir sind im werteprogressiven Sinn aufgefordert, in der Tradition der Menschenrechtskultur Tabus zu halten. Tabu und Aufklärung gehören zusammen« (ebd.). Damit legt sie nicht nur eine Paraphrase, sondern gleichsam eine Verifikation der Freudschen Amalgamierung von Tabu und Moderne vor. Was sich als »Totemlogik des Rechts« bezeichnen läßt, wird an dieser Stelle auch als Movens politischen Handelns deutlich.

14 Hans Kelsen: Reine Rechtslehre, o.O. 1960, S. 5.

15 Ebd., S. 196. 
»muß bei einer Norm enden, die als letzte, höchste vorausgesetzt wird. $«{ }^{16}$ Dieses Begründungsdilemma prägt das moderne Recht; es hält es zugleich transzendent und für die Transzendenz offen. Für die deutsche Verfassung läßt sich sagen, das Grundgesetz nehme durch seine herausgehobene Stellung und Nicht-Antastbarkeit eine solche Funktion als »Grundnorm« wahr. Schließlich setzt es an seinen Anfang mit Artikel 1 (1) eine Klimax, die als basale ethische und soziale Grundnorm des gesamten daran angebundenen Gemeinwesens aufgefaßt werden kann. Daraus ergibt sich eine mögliche Verbindung zur Bedeutung des Tabus in Praktiken des Rechts und der Aufklärung. ${ }^{17}$ Die These von Costas Douzinas, das Recht werde »zur absoluten und transzendenten Kraft der Moderne « erlangt damit erneut Gewicht. ${ }^{18}$

Sofern man annehmen darf, es gäbe bei der Generierung und Implementierung juridischer Gemeinschaften einen animistischen Kontext, bliebe zu klären, wie dieser heute zu spezifizieren wäre und welche Bedeutungsleistungen er vollbringt. Als die Rechtssubjekte kreierende Instanz, rekurriert das Recht selbst auf einen magischen Akt der Verwandlung und läßt aus »Etwas « etwas anderes werden: einen »Jemand «, eine Person. Zur Qualität eines solchen »Jemand« erläutert Robert Spaemann, es handele sich um eine Abhebung des Individuums als Person von der bloßen Zugehörigkeit zur Spezies, die dennoch mit dieser identisch bleibe. Die Grenzziehung, die das Recht dazu vornimmt, und Jemanden aus der Natur des ihn umgebenden Etwas herauslöst, bekräftigt Durkheims Äußerung, auch der soziale Bereich sei der Natur zugehörig, müsse

16 Ebd., S. 197.

17 Unter dem Eindruck jüngerer Debatten um die Legitimität der Folter im Rechtsstaat, hat der Staatsrechtler Ralf Poscher von der »Menschenwürde als Tabu« gesprochen, da die Verhältnismäßigkeits- und Abwägungsdogmatik des Verfassungsrechts die Menschenwürde einem solchen Abwägungsprozeß grundsätzlich entziehe und, wie er in Anlehnung an Max Weber ausführt, für diesen Gegenstand auf eine »wertrationale Betrachtung « umstelle. Dieses Verhalten leite sich daraus ab, daß die »Menschenwürdegarantie des Grundgesetzes nicht die Struktur eines einfachen Verbots, sondern die eines Tabus aufweist« (Ralf Poscher: »Die Würde des Menschen ist unantastbar«, in: Juristen Zeitung, Heft 15/16/2004, S. 758). Mit der Tabuisierung der Menschenwürde greife daher die »moderne Gesellschaft zur rechtlichen Stärkung ihres Fundamentalwerts auf eine archaische Regelungsform zurück « (ebd., S. 759) und entziehe sie somit der Notwendigkeit einer rationalen Begründung. Die das Tabu auszeichnende, »verdeckte Rationalität der Absolutheit des Menschenwürdeschutzes « liege darin, daß sie eine Prophylaxe gegen eine Verrohung der Gesellschaft, besonders der Exekutive, bilde (vgl. ebd., S. 760f.). Wenn Hauke Brunkhorst dagegen einwendet, auch bei der Menschenwürde handele es sich »um eine Norm positiven Rechts «, keineswegs aber um ein Tabu (vgl. Hauke Brunkhorst: Folter vor Recht. Das Elend des repressiven Liberalismus, in: Blätter für deutsche und internationale Politik, Heft 1/2005, S. 80), da sie aus Debatten hervorgehe, solchen weiterhin ausgesetzt, mithin Kulturprodukt sei, dann übersieht er den entscheidenden, von Poscher hervorgehobenen Punkt. Das Tabu ist keineswegs immun gegen Transformationen; diese bedürfen aber nicht allein des Wettstreits der Argumente, sondern insbesondere der Person des ein Opfer darbringenden Tabubrechers, womit eine ungleich wirksamere Hürde gegen die Verletzung substantieller ethischer Codices bereitgestellt sei, als kommunikatives Handeln allein sie jemals aufbieten könnte.

18 Vgl. Costas Douzinas: Die Legalität des Bildes, in: Ludger Schwarte/Christoph Wulf (Hg.): Körper und Recht. Anthropologische Dimensionen der Rechtsphilosophie, München 2003, S. 166. 
aber durch spezifische Praktiken davon abgetrennt werden. Mit dem Recht liegt eine Kulturtechnik vor, die Natur und Kultur (oder: Soziales) voneinander trennt und einen naturlosen, latent transzendent gefaßten Raum generiert, der trotzdem immer in seiner natürlichen Umwelt grundiert bleibt. Bereits im Prozeß der Menschwerdung liegt demnach ein Verwandlungsgeschehen vor, das sich nicht auf den rein biologischen Vorgang der Ontogenesis beschränkt, sondern vor allem auf die sich darin vollziehende Generierung unterschiedlich bewerteter Lebensformen. Indes spricht das Grundgesetz vom Menschen, der Würde besitzt, nicht vom menschlichen Leben, das erst sekundär kontextualisiert wird. Innerhalb der Diskussion um die Legitimität der Biowissenschaften ist dieser Begriff der menschlichen Würde von zentraler Bedeutung, weil er durch die Artikel 1 (1) und 2 (2) Grundgesetz Verfassungsrang erlangt und Rechtskraft entfaltet hat. Der Terminus »menschliche Würde« meint daher nicht nur ein ethisches Prinzip, das ideengeschichtlich und kulturell diese oder jene Gründe für sich geltend machen kann. Es handelt sich vielmehr um ein ethisches Apriori im Verfassungsrang.

Der Begriff der »menschlichen Würde« darf als leitender Topos der deutschen Verfassung gelten; geltendes Recht muß stets in Einklang mit den Prämissen der Menschenwürde stehen. Deren Relevanz für Fragestellungen der Reproduktionsmedizin und Humangenetik hat Günter Dürig bereits in seiner wegweisenden Untersuchung über den Grundrechtssatz von der Menschenwürde hervorgehoben. ${ }^{19}$ Matthias Kettner zufolge konnte die Idee der Menschenwürde schließlich für eine protektive Bioethik so attraktiv werden, als die vorhandenen normativen Ressourcen in der Medizin offenbar nicht hinreichend seien, »um den biomedizinischen Fortschritt so zu regulieren, dass seiner moralischen Integrität wirklich zu trauen wäre. ${ }^{20}$ Aus diesem Grund erfolge der Rückgriff auf die scheinbar finale, freilich auch ganz abstrakte moralische Idee in Gestalt der Menschenwürde. In der Logik des Rechts ist das freilich auch deshalb so, weil Definitionen des Embryos eine Würdigung des werdenden Menschen, der jener auch ist, nach sich ziehen. Indem das Verständnis der Menschenwürde negativ von ihrer Verletzung her gedacht wird, bleibt definitionsoffen, welche Lebensformen exakt in ihrer Lebenswürde verletzt werden können.

Dessen ungeachtet formuliert Dürig generelle Prämissen für die Achtung der Menschenwürde, deren sittlicher Anspruch »gegenüber politischen und rechtlichen Eingriffen des Staates als eigenständig anerkannt, gleichzeitig aber auch im bisherigen individual- und sozialethischen Bereich verrechtlicht worden $\ll$ sei. $^{21}$ Die Menschen-

19 Günter Dürig: Der Grundrechtssatz von der Menschenwürde, in: Abhandlungen des öffentlichen Rechts, Nr. 81, Heft 2/1956, S. 126; 130.

20 Matthias Kettner: Vorwort, in: ders. (Hg.): Biomedizin und Menschenwürde, Frankfurt/M. 2004, S. 10; vgl.a. ders.: Forschungsfreiheit und Menschenwürde am Beispiel der Stammzellforschung, in: Aus Politik und Zeitgeschichte. Beilage zur Wochenzeitung Das Parlament, B 23-24/2004.

21 Günter Dürig: Der Grundrechtssatz von der Menschenwürde, a.a.O., S. 118; vgl.a. Theodor Maunz/Günter Dürig (Hg.): Grundgesetz. Kommentar, Bd. 1, München 2002 (41. Lieferung). Die 42. Lieferung vom Februar 2003 stellt eine gegenüber der als klassisch geltenden Interpretation von Dürig mit dem Kommentar von Matthias Herdegen insbesondere mit Blick auf Problemlagen der Bioethik eine Akzentverschiebung dar, die auf größere Spielräume für die Biowissenschaften zielt. 
würde stelle »einen absoluten, d.h. gegen alle möglichen Angreifer gerichteten Achtungsanspruch « dar; Artikel 1 (1) Grundgesetz sei zur »Basis für ein ganzes Wertesystem « avanciert. ${ }^{22}$ Dürig installiert den Satz von der Menschenwürde als verfassungsrechtliches Leitbild und folgt einem Verständnis des Grundgesetzes, besonders des Artikel 1 (1), als Grundnorm bundesdeutschen Rechts. Der in seiner Auslegung zu Artikel 1 (1) Grundgesetz zum Ausdruck kommende Einfluß Kants auf die bundesdeutsche Rechtstheorie und -praxis ist erheblich, insbesondere wegen der durch ihn ermöglichten Verknüpfung von Recht und Ethik. Ein solcher Ansatz einer ethisch begründeten (an Kant orientierten) rechtlichen Anerkennung scheitert aber Douzinas zufolge am entscheidenden Punkt: »precisely when the expectation has been created that the earlier partial and formal conceptions of law and rights would be transcended by a more and inclusive ethic of care. ${ }^{23}$ Daher stellt sich sowohl die Frage nach der Stiftungsinstanz, die in einer scheinbar »nachmetaphysischen Gesellschaft « (J. Habermas) nicht mehr ohne weiteres numinos übernommen werden kann, als auch das Problem aufgeworfen wird, woran sich der Wert der Würde überhaupt messen läßt.

\section{Performativität des Rechts}

Trotz dieser offensichtlichen Ambivalenzen ist ein Urteil wie das der Rechtswissenschaftler Costas Douzinas und Lynda Nead nach wie vor selten, die das Recht als modernen Erben der Religion bezeichnen, das sich dadurch auszeichne, daß es sich als über allen anderen Diskursen und Praktiken stehenden Metadiskurs präsentiere. ${ }^{24} \mathrm{Da}-$ mit werde auch das Moment einer rechtlichen Bilderproduktion relevant: »Jurisprudence creates an imaginery picture of law as ordered, systematic, closed, coherent and, hermeneutically stable [...]. $\ll^{25}$ Mit dem Recht liegt gewissermaßen eine Struktur vor, aus der sich wirkmächtige Symbolisierungen und Repräsentationen destillieren lassen. Mit ihm kristallisiert sich diejenige Institution heraus, die sowohl Verfahrensweisen für soziale und kulturelle Institutionalisierungspraktiken vorstellt, als auch spezifische Reglements und Verhaltenscodices auflegt, die den Bestand nicht nur des Rechts selbst, sondern einer Vielzahl gesellschaftlicher Institutionen garantieren. Von zentraler Bedeutung in diesem Zusammenhang, so Douzinas, sei die symbolische Repräsentation durch das Recht, worin sich kulturelle Bedeutung und individuelle Vorstellung überschneiden: »As a creation of law and power, the image is unnatural, false; but as the necessary support of our humanity, it is the only truth we have. ${ }^{26}$ Auch die Wahrheit des Rechts ist eine fragile Wahrheit, deren Faktizität auf Modi kultureller Reprä-

22 Günter Dürig: Der Grundrechtssatz von der Menschenwürde, a.a.O., S. 119.

23 Costas Douzinas: Identity, Recognition, Rights or What Can Hegel Teach Us About Human Rights, in: Journal of Law and Society, Vol. 29, No. 3/2002, S. 394f.

24 Vgl. Costas Douzinas/Lynda Nead: Introduction, in: dies. (Hg.): Law and the Image. The Authority of Art and the Aesthetics of Law, Chicago/London 1999, S. 3.

25 Ebd., S. 4.

26 Costas Douzinas: Proposon and Antiproposon. Prolegomena for a Legal Iconology, in: ders./Lynda Need (Hg.): Law and the Image, a.a.O., S. 66f. 
sentationen beruht und Geltung auf diesem Wege erst herstellt. Das Recht kann nicht abgekoppelt von seinen symbolischen, imagologischen, bildlichen sowie semiotischen Aspekten betrachtet werden.

Das Problem der Verbildlichung bleibt ein zentrales Problem in der Bestimmung des Menschen, auch wenn jede Imagologie auf eine Anzahl semiotisch signifikanter Elemente rekurriert. So ist für den »Menschen « in Hinblick auf die bildliche Seite seiner Phänomenologie die frühembryonale Lebensform absolut nicht exklusiv, sondern in diesem Entwicklungsstadium mit der bildlichen Repräsentation nahezu jedes lebendigen Organismus identisch. Lediglich ihre Einbeziehung in die Gesamtheit der Menschheit macht die frühembryonale Lebensform exklusiv, indem sie eine spezifische Gestalt menschlicher Lebensformen und menschlicher Würde repräsentiert. Damit fällt ihr eine Art kultureller Mehrwert zu, der sie von der Existenz als bloße Natur abhebt. Doch genau dies, die Identifizierung der frühembryonalen Lebensform als »Mensch«, die ihr menschliche Würde sowie Schutz durch das Gesetz verleiht, bleibt letztlich vollkommen abstrakt. Es scheint daher nicht abwegig zu sein, von einer anthropotechnischen Qualität des Rechts auszugehen, die sich aus dessen rekursiver Organisation ergibt. In seinen Praktiken zur Bedeutungsgenerierung greift das Recht sowohl auf anthropologische Imaginationen zurück, als es auch maßgeblich zur Konstruktion eines anthropologischen Selbstverständnisses beiträgt: »Offensichtlich verhält es sich so, dass unser Rechtsverständnis unauflöslich an ein bestimmtes Anthropologicum gekoppelt ist. $\ll{ }^{27}$ In diesem Sinne geht Gerald Hartung davon aus, das Recht schaffe sich »seinen Menschen « selbst. ${ }^{28}$ Diesen anthropologisch-konstruktiven Charakter unterstreicht Douzinas und verortet ihn insbesondere im Institut der Menschenrechte. ${ }^{29}$ Die gängige Perspektive auf die Menschenrechte kehrt er um und adressiert diese weniger als schützendes und bewahrendes Instrumentarium für eine vorhandene menschliche Spezies und deren ethische Würde. Vielmehr zeigt er auf, inwieweit es gerade die Menschenrechte sind, die als Instrument für eine Konstruktion von Menschen im Sinne spezifischer Menschenbilder dienen. ${ }^{30}$ Indem sie den Menschen auf eine bestimmte, kulturellen, ideologischen oder politischen Regeln folgende Weise herstellen, stellen Institute wie die Menschenrechte erst Kategorien dafür bereit, kulturell limitierend auf den Menschen zuzugreifen. Ihre protektive Dimension entfalten sie erst aposteriori und für diejenige Form des Menschen, die sie zuvor zumindest herzustellen mithalfen: »Human rights do not belong to humans and do not follow the dicta-

27 Gerald Hartung: Menschenwürde und Lebensschutz. Zur Annäherung von philosophischer Anthropologie und Rechtsdogmatik, in: Ludger Schwarte/Christoph Wulf (Hg.): Körper und Recht, a.a.O., S. 232.

28 Ähnlich bemerkt Jasanoff, das Recht interpretiere heute nicht mehr nur die sozialen Auswirkungen von Wissenschaft und Technologie, sondern konstruiere höchstselbst das soziale Umfeld (»social environment«), worin diese Bedeutung, Gebrauch und Einfluß erführen (vgl. Sheila Jasanoff: Science at the Bar, a.a.O., S. 16).

29 Vgl. Costas Douzinas: Identity, Recognition, Rights, a.a.O.

30 Vgl. zum Menschenbild im Kontext des Rechts: Karl Heinz Auer: Das Menschenbild als rechtsethische Dimension der Jurisprudenz, Wien 2005; Ernst-Wolfgang Böckenförde: Vom Wandel des Menschenbildes im Recht, Münster 2001; Ulrich Becker: Das >Menschenbild des Grundgesetzes< in der Rechtsprechung des Bundesverfassungsgerichts, Berlin 1996. 
tes of humanity; they construct humans. $\ll^{31}$ So gesehen scheint jede Definition darüber, welche Lebensformen unter eine Bestimmung als »Mensch « fallen, sowohl kontingent, als auch von einem kulturellen Imaginären beeinflußt zu sein. ${ }^{32}$

In klarem Gegensatz zu derartigen arbiträren Perspektiven auf die Menschenrechte identifiziert Stephan Rixen die Menschenrechte, durchaus essentiell, vornehmlich als Träger einer ordnenden Kraft innerhalb posttraditioneller Gesellschaften, deren Aufgabe es sei, die sozialen Bedingungen für »normative Homogenität« bereitzustellen. ${ }^{33}$ Gerade das Recht avanciert daher zum Garanten gesellschaftlicher Stabilität; es soll die geeigneten Mittel bereitstellen, um eine kollektiv geteilte Wirklichkeit moralischer Werthaltungen nicht nur anzubieten, sondern auch durchzusetzen. Um diesen Effekt auf der Ebene kulturell wirksamer »symbolischer Formen $\aleph^{34}$ zu erreichen, ist allerdings auch die Einbeziehung bildlich orientierter Imagologien notwendig, womit die von Douzinas sogenannte »Rechtsikonographie« an Bedeutung gewinnt. ${ }^{35}$ In dieser Perspektive haben gerade die deutsche Rechtsprechung und Rechtswissenschaft in den letzten Jahren ein ganzes Arsenal an Techniken und Werkzeugen entwickelt, um frühembryonale Lebensformen, die sowohl rechtlich als auch kulturell und biologisch anthropologisch noch nicht eindeutig bestimmbar sind, in »wirkliche Menschen « zu transformieren. Diese Techniken sind vornehmlich semiotischer Natur. Sie reichen von speziellen Gesetzen wie dem Embryonenschutzgesetz (ESchG) oder dem Stammzellgesetz (StZG), über eine breite rechtswissenschaftliche Diskussion daran anknüpfender Probleme bis hin zu den von Bundesverfassungsgericht (BVerfG) und Bundesgerichtshof (BGH) vorgenommenen Auslegungen bestimmter Gesetze.

Sofern Rechtsprechung und Gesetzgebung bestrebt sind, die Lücke zwischen einer kulturellen Repräsentation der menschlichen Gattung - die im allgemeinen mit dem geborenen Menschen korrespondiert - und deren mikroorganismischen Vorverlaufsformen zu schließen, beabsichtigen sie nichts weniger, als mit einer kulturellen Tradition der Repräsentation des Menschen, wie auch mit der Art und Weise, diesen als geschlossene Gattung zu identifizieren, zu brechen, wie sie sich spätestens seit der Etablierung der Evolutionstheorie durchgesetzt hat. ${ }^{36}$ Das ist möglich, weil es sich bei den Begriffen des Rechts um abstrakte Begriffe handelt, die als »normative Begriffe« (R. Merkel) weder

31 Costas Douzinas: The End(s) of Human Rights, in: Melbourne University Law Review, Vol. 26, No. 2/2002, S. 457.

32 Vgl. zum Konzept eines gesellschaftlichen Imaginären: Cornelius Castoriadis: Gesellschaft als imaginäre Institution, Entwurf einer politischen Philosophie, Frankfurt a.M. 1990.

33 Vgl. Stephan Rixen: Die reprogenetische Diffusion des Körpers: Diffusion der Menschenrechte, in: Ludger Schwarte/Christoph Wulf (Hg.): Körper und Recht, a.a.O., S. 219.

34 Vgl. zum Begriff der symbolischen Form: Ernst Cassirer: Philosophie der symbolischen Formen, 3 Bd.e, Darnstadt 1977.

35 Vgl. Costas Douzinas: Die Legalität des Bildes, in: Ludger Schwarte/Christoph Wulf (Hg.): Körper und Recht, a.a.O., S. 151; vgl. zur Bedeutung des Bildes im Recht auch: Wolfgang Schild: Recht und Körperlichkeit, in: Ludger Schwarte/Christoph Wulf (Hg.): Körper und Recht, a.a.O.

36 Vgl. zur Tradition der Repräsentation des Menschen als geborenem Menschen: Barbara Duden/Jürgen Schlumbohm/Patrice Veit (Hg.): Geschichte des Ungeborenen. Zur Erfahrungs- und Wissenschaftsgeschichte der Schwangerschaft, Göttingen 2002; Gregor Martin Lechner: Maria Gravida. Zum Schwangerschaftsmotiv in der bildenden Kunst, München/ Zürich 1981; Joseph Needham: A History of Embryology, Cambridge 1959. 
durch die Naturwissenschaften noch durch alltagssprachlichen Gebrauch festgelegt sind. ${ }^{37}$ Reinhard Merkel verdeutlicht die kulturhistorische Kontextgebundenheit des Begriffs »Mensch«, wenn er hervorhebt, »alle unsere einfachgesetzlichen Normen«, die diesen Begriff verwenden, bezögen sich lediglich auf den geborenen Menschen, während dem »Embryo« eigene Begrifflichkeiten wie »Ungeborenes« oder »Leibesfrucht « zufielen, die ihn von einer bruchlosen Identität mit dem »Menschen« abzögen. ${ }^{38}$ Auch das Grundgesetz beantworte die Statusfrage embryonaler und frühembryonaler Lebensformen nicht aus sich selbst heraus. ${ }^{39}$ Dazu bedürfe es vielmehr der Interpretation durch die Rechtsprechung des BVerfG als einer $»$ zweite[n] genuine[n] Verfassungsrechtsquelle $\ll .{ }^{40}$ Allen Ansätzen einer protektiven Bioethik, die auch frühembryonale Lebensformen unter den Schutzanspruch des Grundgesetzes stellen wollen und sich auf eine essentielle Anthropologie des Grundgesetzes berufen, erteilt Merkel eine harsche Absage: »Das Grundgesetz selbst schweigt zu der Frage eines subjektiv-grundrechtlichen Status des menschlichen Embryos. ${ }^{41}$ Dagegen setzt Rixen - allerdings im Sinne Merkels gleichfalls unter Rückgriff auf das BVerfG - ein »inklusives Konzept der Menschenwürde«: »Menschenwürde in der Lesart des Gerichts ist voraussetzungslos, sofern es nur um die Würde von Menschen geht. Menschenwürde hat nicht nur den einzelnen Menschen, sondern auch den Menschen als Gattungswesen im Blick «. ${ }^{42}$ Das bedeutet aber in der Tat, ein kulturanthropologisches Konzept zu vertreten, das primär auf die Gesamtheit der Gattung abzielt - und dies nicht nur global phänotypisch, sondern auch ontogenetisch. ${ }^{43}$

Das »inklusive Konzept der Menschenwürde« schließt alle denkbaren menschlichen Lebensformen ein und intendiert damit die Einbeziehung pränataler menschlicher Lebensformen in den Wirkungsbereich des vom Grundgesetz umfaßten Menschenwürdeschutzes. Daß »Probleme der Biowissenschaften immer auch [...] Menschenrechtsprobleme « seien, die zu gleichen Teilen eine rechtliche und eine moralische Dimension beinhalteten, hat Rixen wiederholt herausgestrichen. ${ }^{44}$ In gewisser Weise in Einklang

37 Vgl. Reinhard Merkel: Forschungsobjekt Embryo. Verfassungsrechtliche und ethische Grundlagen der Forschung an menschlichen embryonalen Stammzellen, München 2002, S. 26.

38 Ebd.

39 Allerdings betont John Grace, die meisten zivilisierten Gesellschaften stünden dem ungeborenen Kind gesetzlich geschützte Rechte zu (John Grace: Should the Foetus Have Rights in Law?, a.a.O., S. 58). Das ändert allerdings nichts am uneindeutigen Status embryonaler Lebensformen, deren Rechtsstatus eine Vertretung der Interessen des Embryos nur bedingt zulasse (ebd., S. 61).

40 Reinhard Merkel: Forschungsobjekt Embryo, a.a.O., S. 32.

41 Ebd., S. 34.

42 Stephan Rixen: Verfassungsrecht und Lebensschutz. Verfassungsrechtswissenschaftliche Beobachtungen am Beispiel der Präimplantationsdiagnostik, in: Sigrid Graumann/Katrin Grüber (Hg.): Anerkennung, Ethik und Behinderung, Münster 2005, S. 65f.

43 Der Staatsrechtler Peter Häberle vertritt nachdrücklich genau diese These, Menschenwürde werde mehr und mehr zur »anthropologischen Prämisse des Verfassungsstaates « (Peter Häberle: Verfassungslehre als Kulturwissenschaft, Berlin 1998 S. 350).

44 Vgl. Stephan Rixen: Die reprogenetische Diffusion des Körpers, a.a.O., S. 212. Zu Recht wurde daher gesagt, die biopolitische Debatte erzwinge, »die Grundlagen in einer Rechtsordnung in einer Weise zu artikulieren, die uns die Radikalität dieser Ordnung erst jetzt völlig erschließen lässt « (Tine Stein: Recht und Politik im biotechnischen Zeitalter, in: Deutsche Zeitschrift für Philosophie, Heft 6/2002, S. 868). Was aber nicht heißt, daß in dieser Radikalität auch ein Lösungsmodus für die in dieser Debatte aufgeworfenen Probleme angelegt ist. 
mit der Bildanthropologie Hans Beltings, ${ }^{45}$ geht er davon aus, der Grundrechtsstatus einer Entität sei »im wesentlichen körperbezogener Status - Körperstatus «(St. Rixen), woraus sich die Hervorhebung der Geburt als desjenigen Moments ableite, in dem der Körper sichtbar und daher die Menschenrechte wirksam werden. ${ }^{46}$ Deshalb beabsichtigt Rixen, das anthropologische Argument in kulturhistorischer Perspektive geradezu umzudrehen und gegen eine allzu massive Verwendung des Kontingenzarguments zu kehren. Nicht die Ausdehnung des Menschenwürdeschutzes auf frühembryonale Lebensformen müsse als essentialistisch etikettiert werden, sondern das Insistieren auf der Geburt als einzig möglichem Zeitpunkt für den Beginn von Menschenwürde und Menschenrechten folge einer ihrerseits kontingenten Zuschreibung, die durchaus »kein deskriptiver, sondern ein interpretativer $\mathrm{Akt}$ « sei, zudem gebunden an eine geradezu »suggestive Phänomenalitä̈ « ${ }^{47}$ Die Initiative, mit einer solchen kulturellen Repräsentation des Menschen zu brechen, ging noch von der seinerzeitigen Bundesministerin der Justiz, Herta Däubler-Gmelin (SPD), aus. Sie erklärte, der frühestmögliche Zeitpunkt einer Entwicklung zum Menschen sei auch derjenige von größter Bedeutung für die Frage, wann menschliches Leben und dessen umfänglicher, grundgesetzlich garantierter Schutz einsetze - »Und das ist wohl die Verschmelzung von Samenzelle und Ei. $\ll^{48}$ Allerdings unterscheidet schon ihre Nachfolgerin im Amt, Brigitte Zypries (SPD), zwischen menschlicher Würde, die, wie sie sagt, allen Angehörigen der menschlichen Gattung zukomme und einem Recht auf Leben, das nicht absolut geschützt werden müsse. Zypries formuliert, daß »dem Gesetzgeber bei der Wahrnehmung seines Schutzauftrags für das menschliche Leben ein Spielraum verbleiben muss. « ${ }^{49}$ Damit verbunden ist eine Zurücknahme der von Däubler-Gmelin vertretenen Politik, die Gattung »Mensch« kulturanthropologisch auf frühembryonale Lebensformen auszuweiten. Sofern der Begriff des Menschen eine spezifische, bildliche und semiotische Repräsentation benötigt, um als solcher akzeptiert zu werden, kann es durchaus riskant sein, sich in Richtung einer Änderung respektive einer Transforma-

45 Vgl. Hans Belting: Bild-Anthropologie. Entwürfe für eine Bildwissenschaft, München 2001.

46 Auch Wolfram Höfling hebt die besondere Bedeutung des Körpers nicht nur für die Unversehrtheit des Menschen, sondern auch für dessen Selbstbestimmungsrecht hervor (vgl. Wolfram Höfling: Verfassungsrechtliche Aspekte der Verfügung über menschliche Embryonen und »humanbiologisches Material«, a.a.O., S. 93); zum Verhältnis von Körper und Recht vgl.a.: William MacNeil: Law's Corpus Delicti: The Fantasmatic Body of Rights Discourse, in: Law and Critique, No. 1/1998.

47 Stephan Rixen: Die reprogenetische Diffusion des Körpers, a.a.O., S. 215. Das BVerfG geht davon aus, daß das Menschsein und der Schutz des Einzelnen gegen Angriffe auf dessen Menschenwürde »nicht mit dem Tode « endeten: »Es würde mit dem verfassungsverbürgten Gebot der Unverletzlichkeit der Menschenwürde, das allen Grundrechten zugrunde liegt, unvereinbar sein, wenn der Mensch, dem Würde kraft seines Personseins zukommt, in diesem allgemeinen Achtungsanspruch auch nach seinem Tode herabgewürdigt oder erniedrigt werden dürfte (BVerfGE 30, Nr. 16, 194 - Mephisto-Entscheidung).

48 Herta Däubler-Gmelin: »Selektion ist nicht akzeptabel«, in: Die Zeit, 26. Juli 2001.

49 Rede der Bundesjustizministerin Brigitte Zypries: Vom Zeugen zum Erzeugen? Verfassungsrechtliche und rechtspolitische Fragen der Bioethik, in: Frankfurter Rundschau, vom 30. Oktober 2003. 
tion dieser Repräsentationsform zu engagieren. Frühembryonale Lebensformen - gemessen an ihrer rechtlichen Bedeutung - in vollwertige Menschen zu verwandeln, bedeutet gleichsam die kulturelle Symbolisierung des Menschlichen zu verändern.

\section{Offenes Menschenbild?}

Vor dem nüchternen Hintergrund des von ihm vertretenen Utilitarismus stellt Hoerster daher durchaus die richtige Frage, mit der er ins Zentrum der Problematik zielt: »Ist jenes menschliche Wesen oder Individuum im biologischen Sinn des Wortes, das ohne Zweifel zum Zeitpunkt der Befruchtung zu existieren beginnt, auch bereits im verfassungsrechtlichen Sinn ein Mensch? ${ }^{50}$ Natürlich geht es Hoerster an dieser Stelle um semiotische Aspekte des Begriffs »Mensch«. Er verteidigt einen Begriff des »Menschen«, der sich über eine symbolische Bedeutung legitimiert und verweigert ausdrücklich dessen Ausweitung auf alle denkbaren hybriden Lebensformen der menschlichen Ontogenese. Für ihn gehört der Embryo keineswegs zur kulturellen Gattung »Mensch«, und er macht eine klare Trennung zwischen der biologischen und der kulturellen Spezies. Selbst wenn die frühembryonale Lebensform biologisch gesehen als menschlich bezeichnet werden muß, ist nach Hoerster unbedingt an einer Differenz hinsichtlich einer kulturellen Bezeichnung der Gattung festzuhalten, zu welcher die frühembryonale Lebensform ebensowenig zugehörig wäre, wie der in seiner Entwicklung fortgeschrittene Embryo.

Hingegen hat der Zweite Senat des BVerfG in seinem 1993 ergangenen Urteil zum Schwangerschaftsabbruch festgestellt, entsprechend Artikel 1 und 2 des Deutschen Grundgesetzes müsse menschliches Leben grundsätzlich durch Staat und Gesetz geschützt werden - und »zum menschlichen Leben gehört auch das ungeborene [...]. Menschenwürde kommt schon dem ungeborenen menschlichen Leben $\mathrm{zu}$, nicht erst dem menschlichen Leben nach der Geburt oder bei ausgebildeter Personalität. « ${ }^{51}$ Dies ist ein deutliches Votum dafür, embryonale Lebensformen als vollwertige Menschen anzuerkennen. Das frühere Mitglied des BVerfG, Paul Kirchhof, erklärt sogar, der über das Grundgesetz garantierte Lebensschutz schließe bereits die befruchtete Eizelle ein, die als Substanz der »Entwicklung des Menschen in seiner abschließenden genetischen Prägung und der dadurch begründeten Individualität « anzusehen sei. ${ }^{52}$ Zudem sei es einer der »elementaren Definitionsaufträge des Grundgesetzes «, den Beginn und das Ende des Lebens zu definieren. ${ }^{53}$ Da aber weder eine rechtlich verbindliche Definition dessen existiert, was »Leben« noch was ein »Mensch« ist, schlägt nunmehr die Stunde rechtstheoretischer Interpreten (wie Kirchhof oder Hoerster), um eine rechtlich

50 Norbert Hoerster: Kompromisslösungen zum Menschenrecht des Embryos auf Leben?, in: Juristische Schulung, Heft 6/2003, S. 529.

51 BVerfGE 88, 203, II, 251. Tine Stein spricht davon, die Rechtsgemeinschaft finde »den Menschen in seiner Würde gewissermaßen vor« (Tine Stein: Recht und Politik im biotechnischen Zeitalter, a.a.O., S. 862).

52 Paul Kirchhof: Genforschung und die Freiheit der Wissenschaft, in: Otfried Höffe et al: Gentechnik und Menschenwürde, Köln 2002, S. 21.

53 Ebd., S. 20. 
legitime Imagination des Menschen zu konstruieren. Aus dem Blick gerät allzu oft, daß das BVerfG im selben Urteil auch einräumt, ein zu Fragen der Abtreibung abgegebenes Urteil habe keinen Aussagegehalt in Bezug auf den rechtlichen Status menschlichen Lebens im Augenblick der Fusion von Ei- und Samenzelle. ${ }^{54}$ Das erschwert es, die befürchtete Herabsetzung »der Ungeborenen [...] von Personen zu Gütern « zu beklagen, wenn man sich zugleich darüber im klaren ist, daß eine Verfassungsformel wie die der »Menschenwürde « als »hochgradig unbestimmt « angesehen werden muß. ${ }^{55}$

Tatsächlich trifft weder das Grundgesetz noch das BVerfG eine Aussage über den Zeitpunkt, da menschliches Leben jenseits seiner bloß biologischen Existenz einsetzt. Dies ermöglicht es Hoerster, explizit zwischen dem »Embryo« und dem »Menschen « zu unterscheiden. ${ }^{56}$ Damit schließt er an eine Traditionslinie an, den $»$ Menschen« vermittels seiner Darstellungspraxis überwiegend als geborenen Menschen zu identifizieren. $\mathrm{Da} \beta$ sowohl das Menschenbild Kants als auch das des Grundgesetzes keineswegs »die grundstürzenden Entwicklungen der Humangenetik der letzten 10-15 Jahre « widerspiegeln, hat Wolfgang Graf Vitzthum bereits 1985 hervorgehoben, daraus jedoch nicht die Konsequenz gezogen, den Menschenwürdebegriff selbst einer Revision zu unterziehen. ${ }^{57}$ Statt dessen dehnt er diesen seiner Ansicht nach durch die Humangenetik überholten Begriff auf die neuen biowissenschaftlichen Entitäten aus. ${ }^{58}$ Hier ist bereits unübersehbar, was sich als die katechontische Funktion der Bioethik bezeichnen ließe: über kantianisch legitimierte Begründungsstrategien werden kategorische Bestimmungsleistungen des Menschlichen vorgenommen, die anschließend der Diskussion entzogen werden. Ziel ist es, die Geschlossenheit nicht nur des Begriffs zu wahren, sondern vielmehr die eines daran anschließenden Ordnungssystems sozialer und kultureller Repräsentationen sowie außerdem, sich nicht der Frage aussetzen zu müssen, inwieweit ein an Kant orientiertes Menschenbild mit der biowissenschaftlichen Fraktalisierung kulturanthropologischer Vorannahmen in der Gegenwart kompatibel ist. Die Konsistenz dieses Menschenbilds wird durch dessen Abschließung erreicht.

Insofern innerhalb der rechtswissenschaftlichen Debatte der Begriff eines »offenen Menschenbilds « des Grundgesetzes ins Spiel gebracht wird, sind sich dessen Urheber über das Paradox einer Unternehmung im klaren, die beabsichtigt, »das Undefinierbare « zu definieren, »nämlich den Menschen, dessen >Wesen « nach Absicht der Verfassungsväter und -mütter gerade nicht definiert werden soll. $\aleph^{59}$ Die Offenheit des im Grundgesetz zum Ausdruck gebrachten Menschenbildes steht daher als schützende Geste an dessen Beginn, die den Begriff des Menschen vor ideologischer Instrumentalisierung schützen und in seiner Offenheit zugleich normativ aufwerten soll. Das »offene Menschenbild« des Grundgesetzes soll dadurch realisiert werden, »daß man das

54 Vgl. BVerfGE 88, 203, II, 251.

55 Vgl. Michael Pawlik: Der Staat hat dem Embryo alle Trümpfe genommen, in: FAZ, vom 27. Juni 2001.

56 Vgl. Norbert Hoerster: Ethik des Embryonenschutzes, Stuttgart 2002, S. 96ff.

57 Wolfgang Graf Vitzthum: Die Menschenwürde als Verfassungsbegriff, in: Juristen Zeitung, 40 (1985) 5, S. 208.

58 Vgl. ebd.

59 Stephan Rixen: Die reprogenetische Diffusion des Körpers, a.a.O., S. 222. 
menschliche Individuum als >unfestgestelltes< Wesen in seinem >reinen< leiblichen Gegebensein, in seiner [...] lebendigen Körperlichkeit schützt « - wofür eine kategoriale Reduktion des Menschen auf »allein biologisch-natürliche Maßstäbe« notwendig sei. ${ }^{60}$ In alledem sucht das Konzept vom »offenen Menschenbild « an die philosophische Anthropologie Helmuth Plessners und dessen Ablehnung, das »Wesen« einer lebendigen »Ganzheit « zu definieren, anzuschließen. ${ }^{61}$ Das entscheidende menschliche Merkmal der »Exzentrizität« sei von der Physis abgelöst; »Mensch sein [sei] an keine bestimmte Gestalt gebunden und [könne] daher auch [...] unter mancherlei Gestalt stattfinden. $\ll{ }^{62}$ Dieser Argumentation korrespondiert, daß die Offenheit des Menschenbildes im Sinne von Höfling und Rixen darin besteht, die Existenz als Mensch nicht an spezifische Leistungskriterien zu knüpfen, die erbracht werden müssen, um als Mensch anerkannt zu werden. Bezeichnenderweise ziehen sie daraus aber Plessner ganz entgegengesetzte Schlußfolgerungen: »Nicht mehr als das bloße biologische Lebendigsein des menschlichen Organismus kann danach der Anknüpfungspunkt für die Achtung als Mensch sein. « ${ }^{63}$ Die größtmögliche Offenhaltung des Menschlichen läge demnach in dessen größtmöglicher Reduktion, die es erlaubt, alle diejenigen Lebewesen, die die Form des Menschen repräsentieren, als Menschen auch anzuerkennen. Im Akt der Anerkennung erfolgt beides: die Bestätigung des Individuums als Rechtssubjekt und die Schaffung eines normativen Raums, worin Recht wirksam werden kann. Das schließt, worauf Douzinas hinweist, eine kulturanthropologische Dimension mit ein: in der Moderne sei die Tatsache Menschenrechte zu besitzen, obwohl man diese einfordern müsse, gleichbedeutend damit geworden, ein Mensch zu sein. ${ }^{64}$

Erst im Akt der Anerkennung, der selbst dezidiert und offenkundig nicht rechtlicher, sondern moralischer Art ist, wird das Recht praktisch und insofern auch wirklich. Die Praktiken der Anerkennung bedeuten den kategorialen Durchgang des Rechts durch ein soziales Imaginäres, an dessen Ende die Konstituierung des Rechts als »symbolische Form« (E. Cassirer) steht. In diesem Sinne hat Habermas proklamiert, eine Rechtsordnung müsse »nicht nur garantieren, daß eine jede Person in ihren Rechten von allen übrigen Personen überhaupt anerkannt wird; die reziproke Anerkennung der Rechte eines jeden durch alle muß vielmehr auf Gesetzen beruhen, die insofern legitim sind, als sie jedermann gleiche Freiheiten gewähren [... « ${ }^{65}$ Zwar fungiert das aus der Philosophie Hegels stammende Anerkennungsprinzip als eine Grundlage des Rechts; es selbst besitzt jedoch keine kausale Begründung. An diesem Punkt tendiert das Recht nahezu zwangsläufig zur Metaphysik. Säkulares Recht will allerdings antimetaphysisch strukturiert sein. So läßt sich entweder mit dem modernen Pragmatismus für

60 Wolfram Höfling/Stephan Rixen: Verfassungsfragen der Transplantationsmedizin. Hirntodkriterium und Transplantationsgesetz in der Diskussion, Tübingen 1996, S. 70.

61 Vgl. Helmuth Plessner: Die Stufen des Organischen und der Mensch. Einleitung in die philosophische Anthropologie, Berlin/New York 1975, S. 120.

62 Ebd., S. 293.

63 Vgl. Wolfram Höfling/Stephan Rixen: Verfassungsfragen der Transplantationsmedizin, a.a.O., S. 71.

64 Costas Douzinas: Identity, Recognition, Rights, a.a.O., S. 399.

65 Jürgen Habermas: Faktizität und Geltung, a.a.O., S. 49. 
einen radikalen Relativismus plädieren, der sehr wohl weiß, daß es absolute Prämissen nicht geben kann. ${ }^{66}$ Oder aber es wird mit Hilfe einer neuen Kategorie versucht, diese Leerstelle der Begründung zu substituieren.

Diese letztere Funktion erfüllt in der bioethischen Debatte der Begriff der »Menschenwürde « auf mehreren Ebenen: rechtlich, politisch, philosophisch und anthropologisch. So betont das BVerfG in seinen einschlägigen Urteilen zur Abtreibungsfrage nachdrücklich die »verfassungsrechtliche Pflicht des Staates [...], das ungeborene menschliche Leben zu schützen. « ${ }^{67}$ Daraus leitet das Gericht schließlich ab, »auch für das ungeborene Leben « liege die Würde des Menschseins »im Dasein um seiner selbst willen. ${ }^{68}$ Sind diese Sätze dem zweiten Abtreibungsurteil von 1993 entnommen, so hatte das Gericht schon in seinem ersten Urteil zum Schwangerschaftsabbruch aus dem Jahre 1975 präzisiert, das menschliche Leben stelle die »vitale Basis der Menschenwürde und die Voraussetzung aller anderen Grundrechte « dar. ${ }^{69}$ Letztlich bleibt aber trotz der Feststellung des BVerfG, Menschenwürde komme »schon dem ungeborenen menschlichen Leben zu, nicht erst dem menschlichen Leben nach der Geburt oder bei ausgebildeter Personalität «, ${ }^{70}$ weiterhin umstritten, in welchem Ausmaß der menschliche Embryo in den Schutzbereich von Artikel 1 (1) Grundgesetz einbezogen werden soll. Weil diese Aussage dezidiert im Kontext einer Abtreibungsdiskussion getroffen wurde, die sich um Feten dreht, ist zudem weiterhin unklar, ob ihre Reichweite sich auch auf die in der Bioethik verhandelten frühembryonalen Lebensformen erstreckt.

Von einem einheitlichen Gehalt des Menschenwürdebegriffs kann keine Rede sein. Vielmehr zielt er in dem Sinne, wie Theodor Heuß ihn eine »nicht interpretierte These « genannt hat, ${ }^{71}$ auf eine Kultur fortlaufender Überlegungen, die das Problem des Menschen in Gesellschaft gegenwärtig hält. Eine solche Formel muß nicht pejorativ gemeint sein. Als »nicht interpretierte These « kann Menschenwürde so wenig exklusiv einzelnen Gruppen vorbehalten sein, wie sie auch keine Sicherheit im Sinne einer definitiven Abschließbarkeit bietet oder mit einer nicht interpretierbaren These verwechselt werden darf. »Menschenwürde« bleibt ein symbolisches Kapital im Fluß der kulturellen Entwicklung, das immer neu investiert und verhandelt werden muß. Die Konjunktur der Menschenwürde im Kontext der Bioethik hingegen markiert vor allem einen Verlust an Identifikationssicherheit gegenüber der eigenen Gattung. Vitzthum hat das klar gesehen, als er konstatierte, die Humangenetik berge in sich das Potential, nicht nur die Identität einzelner Individuen, sondern die Grundlagen der ganzen menschlichen Gattung anzutasten: »Die Gefährdung der Menschenwürde schlüge um in eine neue Qualität - in die schicksalhafte Fähigkeit des homo faber, über Identität und Evolution der menschlichen Spezies zu verfügen. ${ }^{72}$ Das bedeutet nicht nur ei-

66 Vgl. dazu Richard Rorty: Kontingenz, Ironie und Solidarität, Frankfurt/M. 1992.

67 BVerfGE 88, 203, II (213).

68 BVerfGE 88, 203, II (252); vgl.a. BverfGE, 69, 375.

69 BVerfGE 39, 1 (42).

70 BVerfGE 88, 203, II (251).

71 Zitiert nach: Ernst Benda: Erprobung der Menschenwürde am Beispiel der Humangenetik, in: Rainer Flöhl (Hg.): Genforschung - Fluch oder Segen?, München 1985, S. 214.

72 Wolfgang Graf Vitzthum: Die Menschenwürde als Verfassungsbegriff, a.a.O., S. 201. 
nen Umschlag in der Zielsetzung der Menschenwürde, weg vom Schutz des Individuums, hin zu einem Institut der Gattung, sondern auch das Einsetzen einer Argumentation, die einer als unausweichlich empfundenen technischen Entwicklung, vorangetrieben von einem »faustischen Drang der Forschung « (J. Isensee), mit der Abschließung normativer Basiskategorien zu begegnen gedenkt. ${ }^{73}$ Positionen wie diese lassen sich als katechontischer Umgang mit dem Begriff der »Menschenwürde« bezeichnen.

In dezidiertem Gegensatz zu explizit bioethischen Positionen hat hingegen jüngst Matthias Herdegen darauf hingewiesen, das Postulat der Menschenwürde enthalte in keiner Weise eine Grundrechtsdeduktion. Interessanter sei das wechselseitige Verhältnis von Menschenwürdegarantie und nachfolgenden Grundrechten, wobei er hervorhebt, Individuum und Gattung seien gleichermaßen vom Grundsatz der Menschenwürde berührt. ${ }^{74}$ Herdegen spezifiziert, bei deren Träger handele es sich »zunächst [um] jede geborene Person kraft Zugehörigkeit zur Spezies >Mensch «. « ${ }^{75}$ Damit erteilt er utilitaristischen Positionen eine Absage; genauso wendet er sich allerdings gegen eine Ausweitung des Menschenwürdeschutzes auf künstlich erzeugte, pränatale mikroorganismische menschliche Lebensformen. ${ }^{76}$ Tatsächlich nimmt Herdegen den eklatanten Schnitt vor, den Menschenwürdeartikel von einem ihm »vorgelagerten geistig-ethischen Inhalt« (E.-W. Böckenförde) abzulösen. Und wirklich zeigt seine Auslegung, daß der Grundrechtsartikel ohne einen solchen Inhalt kaum mehr Ansatzpunkte für normative Richtliniensetzungen aufweist, sondern sich in Rezeptionsexegesen und Deutungsarbeit verliert. Herdegen mißtraut eindeutig dem metaphysischen Gehalt der Rechtsnorm, wo Dürig ihr auf diese Weise sinnstiftende Gültigkeit verschaffen wollte. Das Resultat zeigt aber, daß das Recht ohne den Bezug auf eine Aussage, die von einer notwendig imaginär bleibenden Grundnorm getroffen wird, haltlos wird. Das trifft um so mehr auf einen Begriff wie den der Menschenwürde zu, worin sich ethische, politische und kulturanthropologische Aspekte vermengen. Das Recht als diejenige Anthropotechnik, die jenem Imaginären soziale Kohärenz verleihen soll, wird somit immer mit dem Dilemma konfrontiert bleiben, über eine derartige Kohärenz keine endgültige Aussage treffen zu können, weshalb es sich in bioethischen Auseinandersetzungen einerseits schlecht als schlagendes Argument benutzen läßt und zugleich dafür Sorge tragen muß, für die vorgebrachten Argumente einen Rahmen bereitzustellen, worin sich deren Gültigkeit bestimmen ließe.

73 So etwa Winfried Brohm, der anmahnt, das Recht könne angesichts der sozialen und ethischen Brisanz der Biotechniken nicht abwarten, bis die Techniken zur Anwendungsreife gelangt seien; »vielmehr müssen möglichst dann schon die Normen für ihre Zulässigkeit und ihre Grenzen bereitstehen« (Winfried Brohm: Humanbiotechnik, Eigentum und Menschenwürde, in: Juristische Schulung, Heft 3/1998, S, 197). Auch Isensee versäumt nicht, darauf aufmerksam zu machen, daß nicht etwa die Erlangung genetischen Wissens problematisch sei, sondern dessen "praktische Verwendung « (Josef Isensee: Das entschlüsselte Genom im Verständnis der Verfassung, in: Ludger Honnefelder/Peter Propping (Hg.): Was wissen wir, wenn wir das menschliche Genom kennen?, Köln 2001, S. 231).

74 Vgl. Herdegen, Matthias: Grundgesetzkommentar: Art. 1 Abs. 1, in: Theodor Maunz/Günter Dürig: Grundgesetzkommentar, Band I, Lieferung 43, München 2003, S. 13.

75 Ebd., S. 29.

76 Ebd., S. $32 \mathrm{ff}$. 